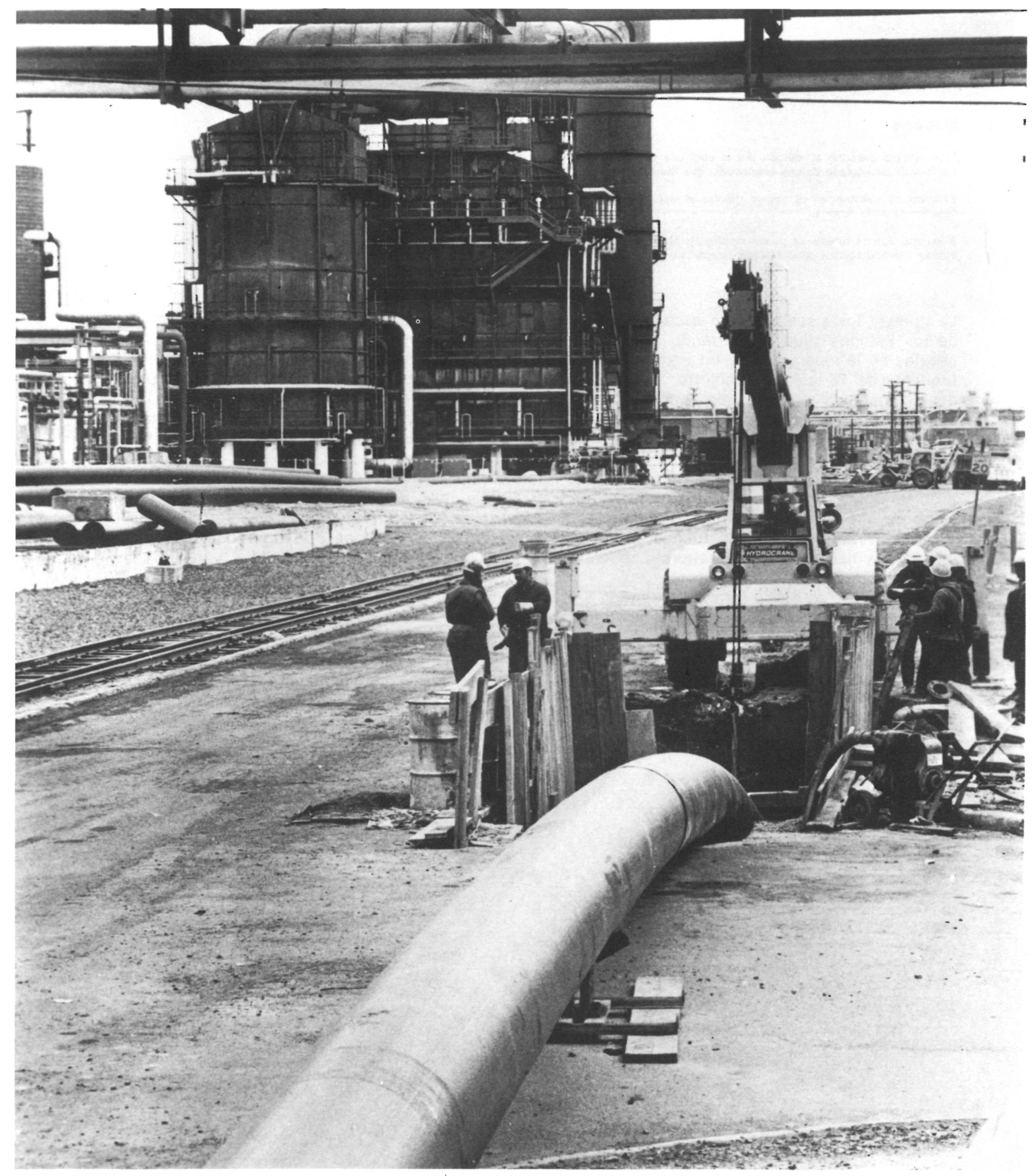

\title{
Ahorro de dinero y de energía mediante la instalación de una conducción termoplástica en una refinería
} EE.UU. 


\section{sinopsis}

Este articulo describe el método por el cual una refineria pudo ahorrarse unos doce millones de pesetas en la operación de repuesto de un tramo muy deteriorado de una conducción que transporta agua salada refrigerante.

Este ahorro substancial se obtuvo gracias al uso de tubos de presión de polietileno, de alta densidad, como revestimiento de la sección deteriorada de la tuberia.

El mismo tipo de luberia se puede emplear también en otras industrias (pastas, papel, procesos quimicos, control de polución, mineria, textil, etcètera) donde existan conducciones desgastadas o deterioradas por corrosión o abrasión.

La primera instalación a gran escala de un conducto termoplástico se hizo en una refinería del este de los Estados Unidos ahorrando a la compañia una cantidad estimada en unos doce millones de pesetas en la reparación de un tramo de $270 \mathrm{~m}$ de longitud de una tubería muy deteriorada de hierro fundido, de $76 \mathrm{~cm}$ de diámetro, que alimenta con agua salada refrigerante a un alambique de destilación. Las mayores contribuciones al ahorro de costes se debieron a la flexibilidad y ligero peso del tubo de presión de alta densidad, lo que permitió la terminación del proyecto de reparación en un tiempo extremadamente corto, con un mínimo de interrupción en las operaciones de la planta.

Hoy, después de 4 años de servicio, la tubería no sólo está operando con una completa ausencia de problemas y con una esperanza de vida de servicio de más de 50 años, sino que proporciona también ahorros de energía importantes en la estación de bombeo del agua salada, pues la superficie interior lisa de la tubería de pelietileno minimiza las pérdidas por rozamiento dentro del conducto y hace más baja la altura cinética total.

La conducción de hierro fundido enterrada a una profundidad de 1,83 $\mathrm{m}$, data de 1915, y forma parte de un viejo sistema de abastecimiento que prové de agua salada al alambique de destilación. Los años de servicio, transportando estas aguas agresivas y una presión de trabajo de $2,45 \mathrm{~kg} / \mathrm{cm}^{2}$, deterioraron seriamente la conducción, necesitando un número creciente de reparaciones. Cuando un tramo de la tubería rompió en 1974 resultó imperativo hacer una operación de repuesto o de rehabilitación. Muestras de la sección afectada de la tubería, así como otras porciones, se analizaron en un laboratorio exterior para ser rigurosamente grafitizadas.

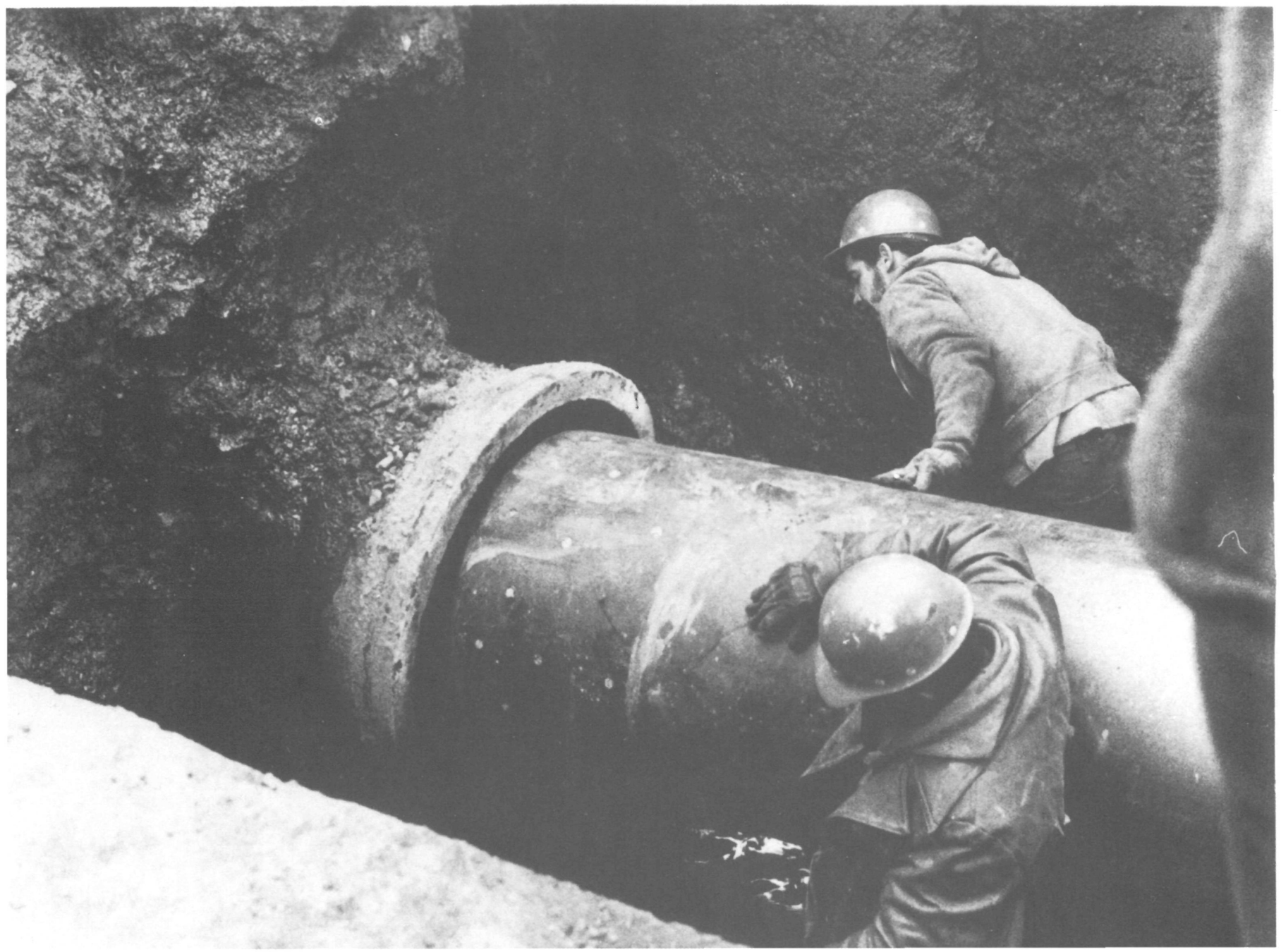




\section{PRIMEROS ANALISIS INGENIERILES QUE LLEVARON A LA ELECCION DE LA TUBERIA DE POLIETILENO}

Se inició inmediatamente un estudio de diversas maneras de reponer el material. La atención se centró primero en un buen repuesto con hierro fundido, pero la existencia de distintas calzadas, ferrocarriles y tuberias con las que se podian producir interferencias hizo a esta alternativa desestimable, desde el punto de vista económico y desde la posible seria interrupción de las operaciones de la planta. El coste de tal sustitución se estimó en unos dieciocho millones de pesetas.

Una alternativa posible para salvar los problemas del alto costo y de las interrupciones de la planta consistía en revestir el interior de la tuberia de hierro fundido existente con una de polietileno de alta densidad. Esta técnica minimiza las necesidades de excavación porque se realiza simplemente insertando el tubo de polietileno en la tuberia deteriorada existente estableciendo, en realidad, una nueva tubería. Comparado con los $270 \mathrm{~m}$ lineales de excavación entibada requeridos, si se hubiese recurrido a la tubería de hierro fundido, el revestimiento sólo necesitó la excavación de $36 \mathrm{~m}$ lineales produciendo un enorme ahorro. Uno de los materiales termoplásticos más apropiados para la aplicación fue el polietileno, que se juzgó el más económico para este tipo de servicio. El desembolso para la instalación de polietileno se presupuestó en 6.000 .000 de pesetas, lo que suponía un ahorro de 12.000 .000 de pesetas sobre la instalación de hierro fundido.

Sin embargo, hasta ahora, no había referencia de un proyecto de revestimiento de esta magnitud y, mucho menos, con una tubería de presión.

Se requirió a la División de Investigación e Ingeniería de la compañía para investigar la viabilidad del uso de la técnica de revestimiento con polietileno de alta densidad y ésta informó que el proyecto básico era factible y que el material satisfacía las normas A.S.T.M. para tales aplicaciones. El informe también anticipó una vida de servicio de más de 50 años con una presión de $5,60 \mathrm{~kg} / \mathrm{cm}^{2} \mathrm{e}$ indicó que la probabilidad de los fallos en la junta era despreciable.

\section{TUBERIA DE POLIETILENO PROVISIONAL INSTALADA EN SEIS DIAS}

Antes de poder comenzar el revestimiento fue necesario instalar un método alternativo de suministro de agua salada refrigerante para que el alambique pudiera seguir sus operaciones normales.

La conducción provisional se hizo con tubería de presión de polietileno de alta densidad, "MLSCO-ppd/305", de $56 \mathrm{~cm}$ de diámetro, que corre a una distancia de $180 \mathrm{~m}$ sobre el suelo hacia el filtro del alambique; se colocó para que pudiese seguir la refrigeración normal de este último. El uso del termoplástico para la citada aplicación produjo también ahorros substanciales. La instalación de una tubería de acero al carbono habria costado 3.250 .000 pesetas, que comparados con los 2.450.000 pesetas de esta tubería de polietileno, da un ahorro del $55 \%$. Además, esta tubería termoplástica es de tal tamaño que permite su uso para una nueva operación de revestimiento de cualquier otro tramo del sistema.

La instalación de las tuberías provisionales de polietileno se realizó a lo largo de 6 días de trabajo, durante una corta parada de las operaciones del alambique. Una vez que la tuberia provisional comenzó a transportar agua salada refrigerante, empezaron las operaciones de revestimiento de la tuberia deteriorada de $76 \mathrm{~cm}$.

\section{TROZOS DE 18 m DE TUBERIA, DE POLIETILENO, SOLDADAS A TOPE PARA FORMAR UN OLEODUCTO}

La tuberia de presión de polietileno utilizada en la instalación de revestimiento fue la "MLSCO-ppd/305", que se suministró en secciones de $18 \mathrm{~m}$. Los diámetros de la tuberia son de 71 centímetros, lo que permitió su inserción en la tubería de $76 \mathrm{~cm}$ de hierro fundido.

Los tramos de $18 \mathrm{~cm}$ se unieron mediante fusión a tope, técnica única para la tubería poliolefina. Los extremos de las tuberías, se unian colocándolos en una máquina especial de fusión a tope, donde se fundían bajo temperatura y presión controladas, con objeto de formar una fuerte junta estanca. Se tardaba menos de una hora en terminar una junta de este tipo, o sea, mucho más rápido que soldando una tubería de acero al carbono o instalando una tubería de hierro fundido con junta de plomo. 


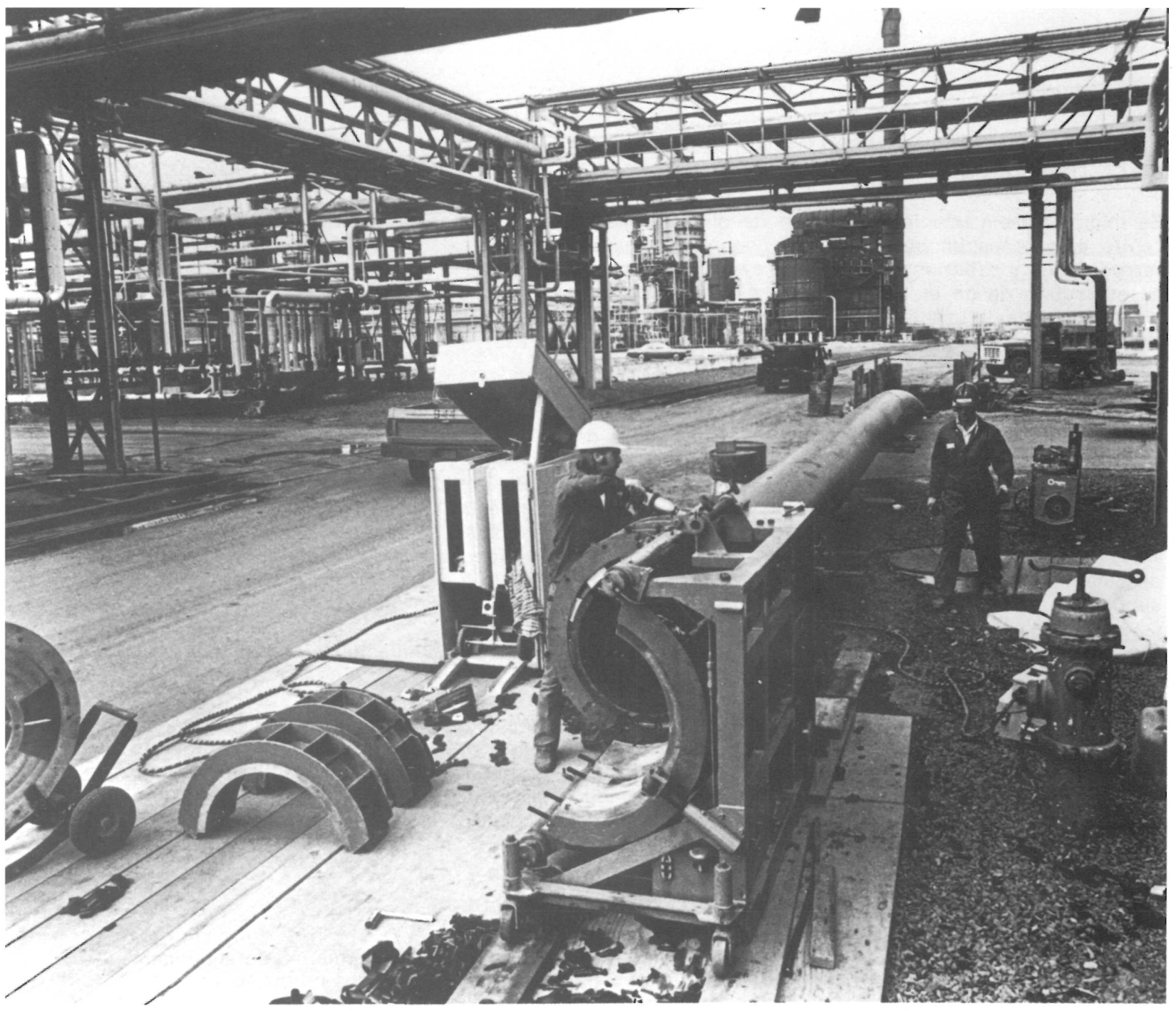

La probabilidad de fallos en la junta se redujo substancialmente gracias a un procedimiento de ensayo rígido que utiliza una técnica ultrasónica desarrollada especialmente para esta instalación por la refinería.

\section{TUBERIA DE POLIETILENO COLOCADA DENTRO DE LA TUBERIA DE HIERRO FUNDIDO}

El acceso a la tuberia enterrada de hierro fundido lo proporcionaron tres excavaciones entibadas que totalizan $36 \mathrm{~m}$ lineales. Una de las excavaciones permitió la inserción de la tuberia de polietileno; la segunda sirvió para la colocación de la tubería de polietileno dentro de la tubería de hierro fundido, y la tercera para la remoción y renovación del tubo deteriorado de hierro fundido por otro más adecuado, hecho de polietileno de alta densidad.

Un lavado completo y una pre-inspección de la tubería de hierro fundido de $76 \mathrm{~cm}$ fueron precisos para asegurar una instalación eficiente de revestimiento. Un taco de acero para limpiar, un instrumento de metal que contiene un cierto número de dientes para escarbar el interior de la tubería, se paseó por ésta aproximadamente cinco veces, hasta asegurar una limpieza completa.

Después de la limpieza, una cámara de televisión en circuito cerrado se introdujo en la tubería y se hizo un "video-tape" de los resultados que proporcionó datos significativos para predecir las dificultades que pudiera haber con la tubería de hierro fundido. 
La tubería de polietileno se insertó en la tuberia de hierro fundido en tres secciones. Un cable se extendió a través de la tuberia de hierro fundido y se fijó a un cabezal de tracción especial, anclado firmemente al extremo de la tubería de polietileno. Un bulldozer, situado encima de la segunda excavación introduce la tuberia de polietileno a través de la tubería de hierro fundido y la coloca en su posición definitiva. Una vez que la tuberia de polietileno se colocó, sus rebordes se atornillaron.

La tuberia que alimenta el alambique se ajustó entre dos secciones y se instaló in situ mediante conexiones de bridas.

La última medida fue un ensayo a $5.600 \mathrm{~kg} / \mathrm{cm}^{2}$, seguido del relleno de las excavaciones, con 10 que se terminó el proyecto de reparación.

Un año más tarde, se hizo una instalación casi idéntica en la misma refineria, con tuberia de polietileno "MLSCO-ppd/305", para los últimos tramos de la tubería de hierro fundido deteriorada.

\section{résumé}

\section{ECONOMIE D'ARGENT ET} L'INSTALLATION D'UNE CONDUITE THERMOPLASTIQUE DANS UNE RAFFINERIE - ETATS UNIS

Dans cet article, l'auteur décrit la méthode permettant une économie d'environ douze millions de pesetas lors de l'opération de rechange d'un tronçon très détérioré d'un conduite d'eau salée réfrigérante.

Cette économie substantielle a été assurée gráce à l'utilisation de tuyaux de pression en polyéthylène à haute densité, comme revêtement de la section détériorée de la conduite.

Le mème type de tuyau peut être égalemen utilisé pour d'autres industries (pâtes, pepler, processus chimiques, contrôle de pollution, mines, textile, etc.) où il existe des conduites usées ou détériorées à cause de la corrosion ou de l'abrasion.

\section{summary}

MONEY AND ENERGY SAVINGS THROUGH THERMOPLASTIC PIPING INSTALLATION IN AN OIL REFINERY - U.S.A.

This article describes the method used by and oil refinery to save some two hundred thousand dollars in the replacement of a
seriously damaged stretch of a pipeline carryseriously damaged stretch
ing salt water refrigerant.

This substantial saving was obtained by installing high density polyethylene pressure pipe as a cover around the damaged pipe section.

The same type of piping can be used in other industries (pulp and paper mills, chomical processes pollution confrol, mining, textile mills, etc.) where worn or abrasion corrosion damaged pipes can be found.
PLASTIC PIPELINES DIVISION M.L. SHELDON PLASTICS CORP NEW YORK zusammenfassung

GELD- UND ENERGIEERSPARNIS DURCH DIE EINRICHTUNG EINER THERMOPLASTISCHEN LEITUNG IN EINER RAFFINERIE - USA

Dieser Artikel beschreibt das Verfahren nach welchem eine Raffinerie beim Aus nach welchem eine Raffinerie beim Auswechsein eines stark beschänigten Rohrielwasser zur Kühliung ca. Zwölf Millionen Poseten einsparen kann.

Dlese bedeutende Ersparnis konnte Dank der Verwendung von Polyäthylen-Druckrohren hoher Dichte erzielt werden, welche als Verkleidung des beschädigten fohrleitungsteiles Einsatz fanden.

Dieselbe Rohrleitunggstype kann auch in anderen Anlagen (Pasten, Papier, chemische Prozesse, Versäuchungskontrolle, Bergbau, Textilindustrie usw.) verwendet werden, in denen abgenutzte oder beschädigte, der Korrosion oder Reibung ausgesetzte Leitungen vorhanden sind. 